\title{
Scalable user selection in FDD massive MIMO
}

\author{
Xing Zhang* ${ }^{*}$ and Ashutosh Sabharwal
}

\section{*Correspondence:}

ee.xing.zhang@gmail.com Department of Electrical and Computer Engineering, Rice University, Houston, USA

\begin{abstract}
User subset selection requires full downlink channel state information to realize effective multi-user beamforming in frequency-division duplexing (FDD) massive multi-input multi-output (MIMO) systems. However, the channel estimation overhead scales with the number of users in FDD systems. In this paper, we propose a novel propagation domain-based user selection scheme, labeled as zero-measurement selection, for FDD massive MIMO systems with the aim of reducing the channel estimation overhead that scales with the number of users. The key idea is to infer downlink user channel norm and inter-user channel correlation from uplink channel in the propagation domain. In zero-measurement selection, the base-station performs downlink user selection before any downlink channel estimation. As a result, the downlink channel estimation overhead for both user selection and beamforming is independent of the total number of users. Then, we evaluate zero-measurement selection with both measured and simulated channels. The results show that zero-measurement selection achieves up to $92.5 \%$ weighted sum rate of genie-aided user selection on the average and scales well with both the number of base-station antennas and the number of users. We also employ simulated channels for further performance validation, and the numerical results yield similar observations as the experimental findings.
\end{abstract}

Keywords: Massive MIMO, FDD, User selection

\section{Introduction}

Massive multi-input multi-output (MIMO) improves wireless communication, which is a key component of next-generation wireless networks [1-13]. A key challenge for operating massive MIMO in frequency-division duplex mode is the large channel overhead in acquiring channel state information. However, a significant fraction of spectrum allocations worldwide are for frequency-division duplexing (FDD) operation. Therefore, there is a significant demand to enable massive MIMO operation in FDD mode.

The scaling challenge of channel measurement in FDD massive MIMO systems is twofold. First is measuring a large number of channels per user. In [14], we proposed directional training, a scalable channel estimation method, that addressed the per-user channel measurement scalability challenge.

The second challenge is measuring the downlink channels for a large number of users to aid in user subset selection for massive MIMO. Note that the massive MIMO-based

(c) The Author(s) 2021. Open Access This article is licensed under a Creative Commons Attribution 4.0 International License, which permits use, sharing, adaptation, distribution and reproduction in any medium or format, as long as you give appropriate credit to the original author(s) and the source, provide a link to the Creative Commons licence, and indicate if changes were made. The images or other third party material in this article are included in the article's Creative Commons licence, unless indicated otherwise in a credit line to the material. If material is not included in the article's Creative Commons licence and your intended use is not permitted by statutory regulation or exceeds the permitted use, you will need to obtain permission directly from the copyright holder. To view a copy of this licence, visit http:// creativecommons.org/licenses/by/4.0/. 
system will likely support an order of magnitude more users per time-frequency slot compared to past generation systems. The state-of-the-art user subset selection methods [15-27] require full downlink channel state information. Even with directional training, the downlink channel measurement overhead scales with the number of users. Meanwhile, the number of users considered for user selection can be much smaller than the number of selected users for downlink beamforming. Therefore, a scalable user selection scheme is needed for FDD massive MIMO. To achieve graceful scalability with the number of users, the challenge is to perform effective user selection before any downlink channel estimation.

In this paper, to reduce the large downlink channel estimation overhead that scales with the number of users in FDD massive MIMO systems, we develop a novel propagation domain-based user selection scheme, labeled as zero-measurement selection. As the name suggests, zero-measurement selection lets the base-station perform user selection without any downlink channel estimation. Zero-measurement selection is driven by the experimental findings that the downlink inter-user channel correlation and user channel norm can be reliably inferred from the uplink channel information in the propagation domain. Overall, our main contributions in this paper are as follows:

1 Based on the observed channel low dimensionality and partial reciprocity in the propagation domain, we investigate the potential of inferring downlink user channel norm and inter-user channel correlation from uplink channel propagation domain information. For the downlink channel norm, we show that the inferred downlink channel norm from uplink channel measurements leads to only about $15 \%$ normalized error. For the downlink inter-user channel correlation, we find out that the estimated inter-user channel correlation based on uplink information in the propagation domain, including paths angles and amplitudes, brings in only about $10 \%$ normalized error.

2 Driven by experimental findings that both uplink inferred downlink inter-user channel correlation and uplink inferred user channel norm provide good approximation with small errors, we develop zero-measurement selection for FDD massive MIMO systems. The key idea of zero-measurement selection is to select users based on inferred downlink inter-user channel correlation and user channel norm from free uplink channel information in the propagation domain. Therefore, no downlink channel estimation overhead is incurred in the zero-measurement selection. Zeromeasurement selection is developed in the well-known proportional-fair form. There are three main steps in zero-measurement selection. First, we extract uplink propagation domain dominant angles and amplitudes from uplink channel and construct downlink channel propagation domain for each user, respectively. Second, during each round of selection, we calculate users' orthogonal component to selected users channel space. Third, we select the user with the largest estimated weighted rate based on the orthogonal component. The output of zero-measurement selection is the selected user set that can be then used by downlink multi-user beamforming methods.

3 To evaluate zero-measurement selection, we use both measured and simulated channels. Our measurement data-based results show that zero-measurement selection 
achieves up to $92.5 \%$ of the weighted sum rate of genie-aided user selection on average when a 64-antenna base-station selects 8 users out of 100 users for downlink beamforming. We then examine the performance scalability with both the number of base-station antennas and the number of users for zero-measurement selection with both measured channels and 3GPP-based simulated channels. The numerical results show that zero-measurement selection scales well, from average $90 \%$ weighted sum rate when a 64-antenna base-station selects 8 users out of 100 users for downlink beamforming, to average $97 \%$ weighted sum rate when a 256 -antenna base-station selects 32 users out of 100 users for downlink beamforming; all comparisons are made with respect to the genie-aided user selection.

The outline for the rest of the paper is as follows: Section 2.1 formulates the research problem for user selection. Section 2.2 illustrates the experimental findings that downlink inter-user channel correlation and user channel norm can be effectively inferred from free uplink channel information in the propagation domain. Section 2.4 describes the proposed zero-measurement selection. Section 3 provides experimental and numerical evaluation of zero-measurement selection. Finally, Section 4 concludes this paper.

\section{Methods}

\subsection{Problem formulation}

We consider the single-cell FDD massive MIMO system, where an $M$-antenna base-station serves $N$ single-antenna ${ }^{1}$ users indexed on the set $\{1,2, \ldots, N\}$. During each time slot, the base-station selects $K<N$ users and performs downlink multi-user beamforming for data transmission to the selected $K$ users. The selected user set index is denoted as $\mathcal{S}=\left\{s_{1}, s_{2}, \ldots, s_{K}\right\}$, where $\mathcal{S} \subset\{1,2, \ldots, N\}$. For the selected $K$ users, with downlink channels denoted as $\mathbf{H}_{\mathcal{S}} \in \mathbb{C}^{K \times M}$, the received signal at users-side $\mathbf{y}_{\mathcal{S}} \in \mathbb{C}^{K}$ can be modeled as:

$$
\mathbf{y}_{\mathcal{S}}=\mathbf{H}_{\mathcal{S}} \mathbf{W}_{\mathcal{S}} \mathbf{x}_{\mathcal{S}}+\mathbf{n}_{\mathcal{S}}
$$

where $\mathbf{x}_{\mathcal{S}} \in \mathbb{C}^{K}$ is the transmitted signals vector, $\mathbf{W}_{\mathcal{S}} \in \mathbb{C}^{M \times K}$ is the beamforming weights matrix based on zero-forcing beamforming, and $\mathbf{n}_{\mathcal{S}} \in \mathbb{C}^{K}$ is the additive noise with elements that follow standard complex Gaussian distribution.

When selecting users to be part of set $\mathcal{S}$ for downlink beamforming, different optimization goals can be considered, e.g., maximizing sum rate or minimizing delay. One wellknown fair user selection scheme is proportional-fair user selection, which maximizes the following weighted sum rate to find the best user set $\mathcal{S}$

$$
\max _{\mathcal{S}, \mu(t)} \sum_{s_{k} \in \mathcal{S}} \mu_{s_{k}}(t) R_{s_{k}}(t)
$$

where $\mu_{s_{k}}(t)$ is the weight of user $s_{k}$ in time slot $t$, which is known at the base-station and can be determined by average throughput in the previous time window [15] or data queue length [16]. $R_{s_{k}}(t)$ is the rate of user $s_{k}$ in time slot $t$. To find the user set that

\footnotetext{
${ }^{1}$ The scheme proposed here can be extended to multi-antenna users scenario, but due to experiment data limitation, we mainly focus on single-antenna users scenario.
} 
maximizes the weighted sum rate in the above equation prior to downlink data transmission, downlink channel state information (CSI) of all the $N$ users at the base-station side is necessary to estimate the weighted rate for each user during selection. However, to obtain downlink CSI, the overhead scales with the number of users $N$, which can be very large (e.g., 100s of users are common in a single cell). To reduce the downlink channel estimation overhead and make user selection scale with the number of users, we seek to perform downlink user selection without any downlink channel training. One possible direction is to investigate the feasibility of inferring partial downlink channel information from available information at the base-station side. Towards that end, we ask two questions:

Q1 What partial downlink channel information can we infer from uplink channel estimates, considering that full channel reciprocity does not hold in FDD mode?

Q2 Can the partial knowledge (Q1) be used effectively for user selection by the base station?

We first answer Question 1 in Section 2.2 and investigate the effectiveness of inferring downlink inter-user channel correlation and user channel norm from uplink channel information in the propagation domain based on measured channel dataset. Then, to answer Question 2, we propose a scalable user selection scheme that lets the base station perform select users based on the inferred downlink inter-user channel correlation and user channel norm from uplink.

\subsection{Experimental findings on estimating downlink channel properties from uplink}

In this section, we answer the first question posed above, on what partial downlink channel information can be inferred from uplink channel information? The key information required for effective user selection includes user channel norm and inter-user channel correlation. Therefore, we investigate the possibility of inferring downlink user channel norm and inter-user channel correlation from uplink channel information, such that no additional downlink channel estimation is needed for user selection.

\subsection{FDD massive MIMO channels in propagation domain}

In propagation domain, full downlink channel of $n$-th user can be represented with multiple paths as

$$
\mathbf{h}_{n}=\sum_{l=1}^{L_{n}} \beta_{n l} \mathbf{a}_{f}\left(\theta_{n l}, \varphi_{n l}\right),
$$

where the channel consists of $L_{n}$ paths, and the $l$-th path has complex coefficient $\beta_{n l}$ and angle of departure with elevation as $\theta_{n l}$ and azimuth as $\varphi_{n l}$. The array response vector $\mathbf{a}_{f}(\theta, \varphi)$ of an $M$-antenna uniform plane array consisting of $M_{r}$ rows and $M_{c}$ columns is defined as: 


$$
\mathbf{a}_{f}(\theta, \varphi)=\left[\begin{array}{c}
1 \\
e^{j \frac{2 \pi}{\lambda} d \sin \theta \cos \varphi} \\
\vdots \\
e^{j \frac{2 \pi}{\lambda} d\left(\left(M_{r}-1\right) \cos \theta+\left(M_{c}-1\right) \sin \theta \cos \varphi\right)}
\end{array}\right],
$$

where $f$ is the signal frequency, $\lambda$ is the signal wavelength, $d$ is the antenna spacing, $\theta$ is the elevation angle and $\varphi$ is the azimuth angle.

Similarly, full uplink channel can also be characterized in propagation domain. While uplink and downlink full channel reciprocity does not hold in FDD mode, in our previous work [14], we found out that uplink and downlink channels share approximately the same low-dimensional propagation space. Thus, in this paper, we examine the potential of inferring downlink inter-user channel correlation and user channel norm from uplink channel propagation domain information. Here we employ our measured FDD massive MIMO channels [14], with all the details explained therein. Overall, the channel dataset includes FDD massive MIMO channels corresponding to 21 non-line-of-sight and 4 line-of-sight user locations, and the base-station is equipped with an 8-row 8-column uniform plane array. For each base-station and user pair, two 20-MHz wide-bands channels, each with 52 OFDM subcarriers and separated by about $72 \mathrm{MHz}$, are measured across around 5000 time frames.

\subsubsection{Inferring downlink channel in propagation domain}

Starting from (3), full downlink channel in propagation domain can be further extended as:

$$
\begin{aligned}
\mathbf{h}_{n} & =\sum_{l=1}^{L_{n}} \beta_{n l} \mathbf{a}\left(\theta_{n l}, \varphi_{n l}\right) \\
& =\sum_{l=1}^{L_{\mathrm{Dn}}} \beta_{\mathrm{Dnl}} \mathbf{a}\left(\theta_{\mathrm{Dnl}}, \varphi_{\mathrm{Dnl}}\right)+\mathbf{e}_{\mathrm{an}} \\
& =\sum_{l=1}^{\hat{L}_{\mathrm{Dn}}} \hat{\beta}_{\mathrm{Dnl}} \mathbf{a}\left(\hat{\theta}_{\mathrm{Dnl}}, \hat{\varphi}_{\mathrm{Dnl}}\right)+\mathbf{e}_{\mathrm{mn}}+\mathbf{e}_{\mathrm{an}}
\end{aligned}
$$

where downlink channel can be approximated with $L_{\mathrm{Dn}}$ dominant paths with largest power, and the $l$-th dominant path has complex coefficient $\beta_{\text {Dnl }}$ and angle of departure with elevation as $\theta_{\mathrm{Dnl}}$ and azimuth as $\varphi_{\mathrm{Dnl}} . \mathbf{e}_{\mathrm{an}}$ denotes the corresponding dominant path approximation error. Also, using estimated $\hat{L}_{\text {Dn }}$ paths with complex coefficients $\hat{\beta}_{\text {Dnl }}$ and angle of departures with elevation as $\hat{\theta}_{\text {Dnl }}$ and azimuth as $\hat{\varphi}_{\text {Dnl }}$ to construct downlink channel will result in an additional estimation error $\mathbf{e}_{\mathrm{mn}}$.

Next, to estimate downlink channel in propagation domain, we need to first estimate dominant angles $\left(\hat{\theta}_{\text {Dnl }}, \hat{\varphi}_{\text {Dnl }}\right), l=1,2, \ldots, \hat{L}_{\text {Dn }}$ and corresponding complex coefficient $\hat{\beta}_{\text {Dnl }}, l=1,2, \ldots, \hat{L}_{\mathrm{Dn}}$. Using our results in [14], we extract dominant angles and corresponding amplitudes from the uplink channel estimates. However, the phase information is not known, so full downlink channel state information cannot be inferred from the uplink estimates. But we observed that estimated dominant angle vectors of one user are approximately orthogonal with each other. Therefore, even without phase 


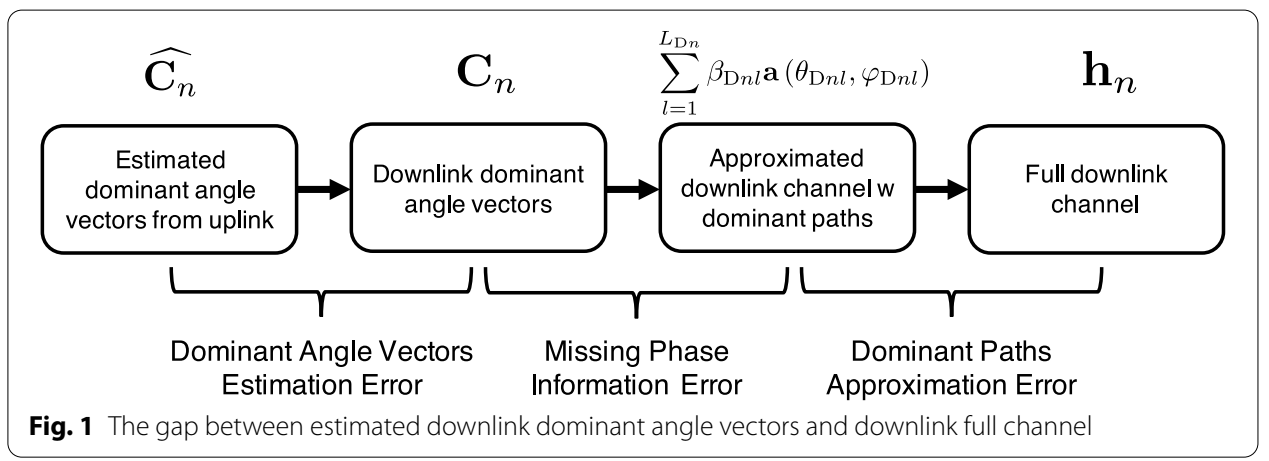

information, we aim to use the estimated dominant angles and corresponding amplitudes from uplink channel to infer per-user channel norm and inter-user channel correlation instead.

Using estimated dominant angles and corresponding amplitudes from uplink channel, we denote estimated downlink dominant angle vectors as:

$$
\widehat{\mathbf{C}_{n}}=\left[\left|\hat{\beta}_{\mathrm{Un} 1}\right| \mathbf{a}\left(\hat{\theta}_{\mathrm{Un} 1}, \hat{\varphi}_{\mathrm{Un} 1}\right) \cdots\left|\hat{\beta}_{\mathrm{Un} \mathrm{Q}_{\mathrm{Un}}}\right| \mathbf{a}\left(\hat{\theta}_{\mathrm{Un} \mathrm{U}_{\mathrm{Un}}}, \hat{\varphi}_{\mathrm{Un}} \mathrm{Q}_{\mathrm{Un}}\right)\right]
$$

where $\left(\hat{\theta}_{\mathrm{Un}}, \hat{\varphi}_{\mathrm{Unl}}\right), l=1,2, \ldots, \hat{L}_{\mathrm{Un}}$ are estimated dominant angles from uplink channel employing the well-known MUSIC estimator with spatial smoothing [28], and $\left|\hat{\beta}_{\text {Unl }}\right|, l=1,2, \ldots, \hat{L}_{\text {Un }}$ are the corresponding estimated amplitudes obtained using leastsquare estimator, with details shown in [14].

We aim to estimate downlink user channel norm and inter-user channel correlation from estimated angle space vectors $\widehat{\mathbf{C}_{n}}$. To evaluate the effectiveness of estimating downlink user channel norm and inter-user channel correlation from uplink channel, we employ our measured FDD massive MIMO channels [14]. Comparing downlink channel $\mathbf{h}_{n}$ and estimated dominant angle vectors $\widehat{\mathbf{C}_{n}}$ from uplink channel, as shown in Fig. 1, there are three sources of error that can affect the estimation performance:

1 Dominant Paths Approximation Error: The approximate error $\mathbf{e}_{\text {an }}$ will lead to the performance gap between full downlink $\mathbf{h}_{n}$ and approximated downlink channel with downlink dominant angles as shown in Eq. 5.

2 Missing Phase Information Error: Without the phase information, we cannot obtain full downlink channel from estimated dominant angle vectors. Thus, the missing phase information will also affect estimation accuracy.

3 Dominant Angle Vectors Estimation Error: Downlink dominant angles and amplitudes are estimated from uplink channel; as a result, the dominant angle vectors estimation error will also degrade the estimation performance.

\subsubsection{Downlink user channel norm estimation}

User channel norm is one of the key information required for effective user selection. Thus, first, we evaluate the effectiveness of estimating downlink user channel norm from estimated angle space vectors set $\widehat{\mathbf{C}_{n}}$, which is shown in Eq. 6. We estimate downlink user channel norm of $n$-th user as follows 


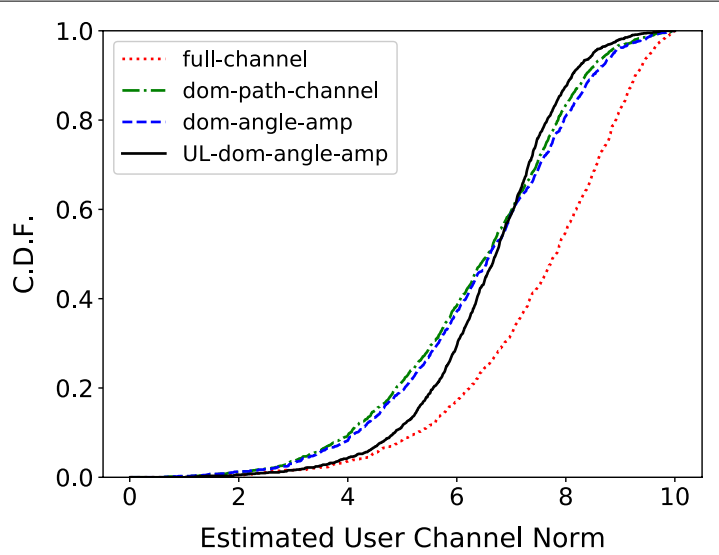

Fig. 2 The cumulative distribution function of estimated downlink channel norm for all measured channels. This includes estimations by utilizing full downlink channel (labeled as full-channel), approximated downlink channel with downlink dominant angles (labeled as dom-path-channel), downlink dominant angles and amplitudes (labeled as dom-angle-amp), and estimated downlink dominant angles and amplitudes from uplink channel (labeled as UL-dom-angle-amp), respectively

$$
\left\|\hat{\mathbf{h}}_{n}\right\|_{2}=\left\|\sum_{l=1}^{\hat{L}_{\mathrm{Un}}} \hat{\beta}_{\mathrm{Unl}} \mathbf{a}\left(\hat{\theta}_{\mathrm{Unl}}, \hat{\varphi}_{\mathrm{Unl}}\right)\right\|_{2} \approx\left\|\widehat{\mathbf{C}}_{n}\right\|_{\mathrm{F}},
$$

where $\|\cdot\|_{2}$ denotes $l_{2}$-norm and $\|\cdot\|_{\mathrm{F}}$ Frobenius norm. The approximation is based on the observation that estimated dominant angle vectors of one user are approximately orthogonal with each other, i.e., $\left|\mathbf{a}\left(\hat{\theta}_{\text {Uni }}, \hat{\varphi}_{\text {Uni }}\right)^{H} \mathbf{a}\left(\hat{\theta}_{\text {Unj }}, \hat{\varphi}_{\text {Unj }}\right)\right| \approx 0, \forall i \neq j$. And the resulting error by the approximation is characterized as Missing Phase Information Error explained above. The cumulative distribution function of estimated downlink user channel norm is shown in Fig. 2.

Finding 1 - Estimated Downlink Channel Norms From Uplink Leads to Small Error: From Fig. 2, we observe that utilizing estimated angle vectors from uplink channel, including dominant angles and amplitudes, to estimate downlink user channel norm, resulting in small error ranging from 0 to $15 \%$.

Explanation for Finding 1: To understand the gap between uplink inferred downlink user channel norm and the actual one, we consider two more ways to estimate downlink user channel norm, including utilizing approximated downlink channel with downlink dominant angles and utilizing downlink dominant angles and amplitudes, to understand the aforementioned three different effects. First, approximation error effect causes error up to $15 \%$ error. Second, missing phase information effect leads to negligible error. Third, uplink/downlink angles and amplitudes mismatch effect results in up to $5 \%$ error. Thus, dominant paths approximation error effect is the dominant one that causes the estimation error. Also, channel norm inferred from dominant angles and amplitudes tends to be smaller due to the fact that dominant angles only capture part of downlink channel power. 


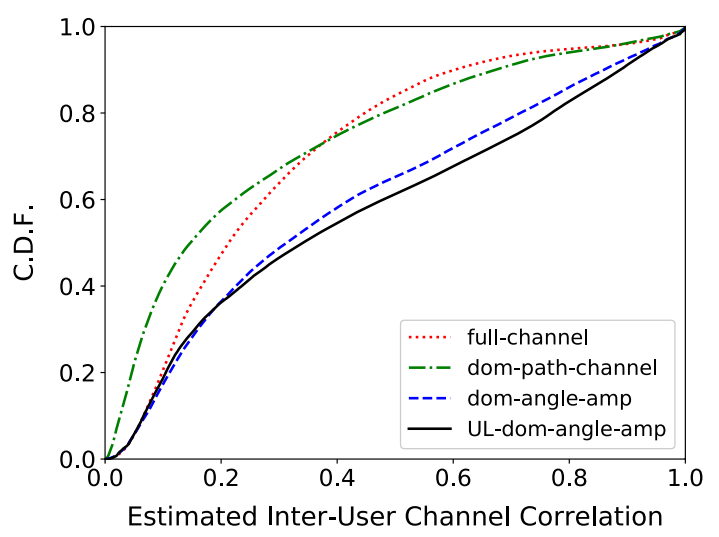

Fig. 3 The cumulative distribution function of estimated downlink inter-user channel correlation for all measured channels. This includes estimations by utilizing full downlink channel (labeled as full-channel), approximated downlink channel with downlink dominant angles (labeled as dom-path-channel), downlink dominant angles and amplitudes (labeled as dom-angle-amp), and uplink downlink dominant angles and amplitudes (labeled as UL-dom-angle-amp), respectively

\subsubsection{Downlink inter-user channel correlation estimation}

Inter-user channel correlation is the other one of the key information required for effective user selection. Thus, we evaluate the effectiveness of estimating downlink inter-user channel correlation from estimated angle space vectors set $\widehat{\mathbf{C}_{n}}$ in Eq. 6 .

Consider users $n$ and $p$. We denote their downlink channels as $\mathbf{h}_{n}$ and $\mathbf{h}_{p}$, respectively. The downlink inter-user channel correlation is formulated as the inner product of their channel vectors

$$
c\left(\mathbf{h}_{n}, \mathbf{h}_{p}\right)=\frac{\left\|\mathbf{h}_{n}^{H} \mathbf{h}_{p}\right\|_{2}}{\left\|\mathbf{h}_{n}\right\|_{2}\left\|\mathbf{h}_{p}\right\|_{2}} .
$$

As the channel correlation can directly affect multi-user beamforming rate, it is one of the key information required for effective user selection.

Then, we aim to estimate the downlink inter-user channel correlation utilizing uplink dominant angles and amplitudes defined in Eq. 6. The estimated downlink inter-user channel correlation is formulated as:

$$
\hat{c}\left(\mathbf{h}_{n}, \mathbf{h}_{p}\right)=c\left(\widehat{\mathbf{C}}_{n}, \widehat{\mathbf{C}}_{p}\right)=\frac{\left\|\widehat{\mathbf{C}}_{n}^{H} \widehat{\mathbf{C}}_{p}\right\|_{\mathrm{F}}}{\left\|\widehat{\mathbf{C}}_{n}\right\|_{\mathrm{F}}\left\|\widehat{\mathbf{C}}_{p}\right\|_{\mathrm{F}}} .
$$

The cumulative distribution function of estimated downlink inter-user channel correlation is shown in Fig. 3.

Finding 2 - Estimated Downlink Inter-user Channel Correlation From Uplink Leads to Small Error: From Fig. 3, we observe that utilizing uplink propagation domain information, including dominant angles and amplitudes, to estimate downlink interuser channel correlation brings in error range from 0 to 0.3 in terms of channel correlation estimation error.

Explanation for Finding 2: Missing phase information error is the dominant contribution to inter-user channel correlation estimation error, causing up to 0.3 correlation 
gap. This is mainly due to the fact that channel vector-constructed space is the subspace of dominant angles response vectors-constructed space.

\subsection{Proposed scheme: zero-measurement selection}

In this section, we answer the second question - Can the base-station perform effective user selection based on partial downlink channel information that is inferred from uplink only? Inspired by the finding that downlink inter-user channel correlation and user channel norm can be effectively inferred from free uplink channel information in the propagation domain, we propose a scalable user selection scheme, labeled as zero-measurement selection for FDD massive MIMO systems. As the name suggests, zero-measurement selection lets the base-station select users for downlink multi-user beamforming before any downlink channel estimation and thus avoid the channel estimation overhead that scales with the number of users. We first provide an overview of the proposed zero-measurement selection scheme, followed by details of each step therein.

\subsubsection{Zero-measurement selection overview}

We develop zero-measurement selection to achieve proportional-fair selection. As described in Section 2.1, we consider the single-cell FDD massive MIMO system, where an $M$-antenna base-station selects $K$ out of $N$ users in each time slot to perform multiuser beamforming. The goal of zero-measurement selection is to solve:

$$
\mathcal{S}=\arg \max _{\mathcal{S}} \sum_{s_{k} \in \mathcal{S}} \mu_{s_{k}}(t) R_{s_{k}}(t) .
$$

The key to solving the above equation is to estimate the rate for each user during selection. When full downlink CSI of all the $N$ users is available at the base station, optimal selection can be obtained using exhaustive search and search all possible user set, to find the one that maximizes the weighted sum rate. In [18], the authors proposed a low complexity greedy type scheme that selects users based on inter-user channel correlation and user channel norm; here we label the scheme as full-channel selection. However, as discussed in Section 2.1, CSI of all the $N$ users is required and large downlink estimation overhead that scales with the number of users $N O(N)$ incurs to obtain downlink CSI.

In contrast, zero-measurement selection does not require any downlink channel estimation. Thus, zero overhead incurs during user selection and only $O(K)$, where $K$ is the number of selected users, incurs to obtain downlink CSI of the selected $K$ users for the subsequent downlink beamforming usage. And the goal is zero-measurement selection to achieve performance close to full-channel selection.

Overall, there are three main steps in zero-measurement selection, as shown in Fig. 4. First, extract users' uplink propagation domain information, including dominant angles and amplitudes. Second, during each round of selection, calculate users' orthogonal component to selected user channel space. Third, select the user with the larger weighted sum rate estimated from the orthogonal component. Next, we present the details of each step. 


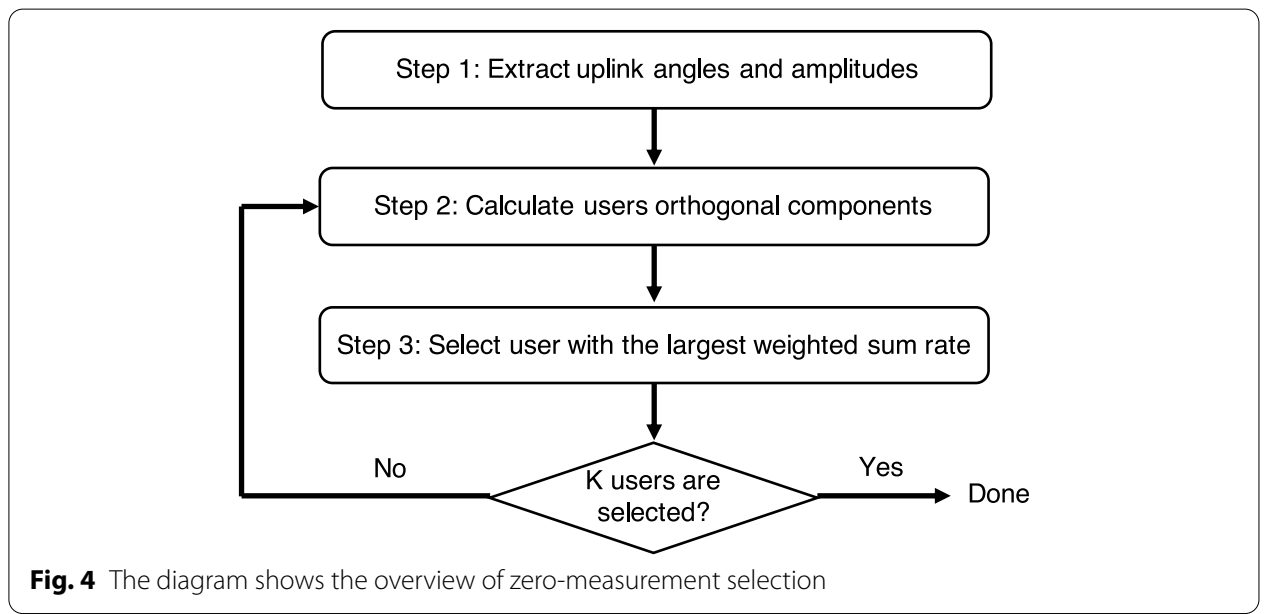

\subsubsection{Zero-measurement selection details}

First, in step 1, extract uplink propagation domain information, including dominant angles and amplitudes. We assume that free estimated uplink CSI is available at the base station $^{2}$ and denote the uplink CSI as $\hat{\mathbf{h}}_{\mathrm{Un}}, n=1,2, \ldots, N$. We employ the well-known MUSIC estimator with spatial smoothing [28] to estimate dominant path angles and least-square estimator to estimate the corresponding dominant path amplitudes. All the estimation details are shown in Section 2.2. The estimated dominant angles of the $n$-th user are denoted as $\left(\hat{\theta}_{\mathrm{Unl}}, \hat{\varphi}_{\mathrm{Unl}}\right), l=1,2, \ldots, \hat{L}_{\mathrm{Un}}$ and the estimated dominant path amplitudes are denoted as $\left\|\hat{\beta}_{\mathrm{Unl}}\right\|, l=1,2, \ldots, \hat{L}_{\mathrm{Un}}$, where $\hat{L}_{\mathrm{Un}}$ is the estimated number of uplink dominant paths. Then, we construct estimated downlink dominant angle vectors $\widehat{\mathbf{C}}_{n}$ using definition in Eq. 6.

Second, in Step 2, calculate the channel orthogonal component to selected user channel space. The selected channel space is denoted as $\hat{\mathbf{S}} \in \mathbb{C}^{M \times M}$ and initialized as $\hat{\mathbf{S}}=\mathbf{0}_{M \times M}$. For the users to be selected, we calculate the channel orthogonal component of $n$-th user as

$$
\widehat{\mathbf{D}}_{n}=\widehat{\mathbf{C}}_{n}(\mathbf{I}-\widehat{\mathbf{S}}) .
$$

Third, in Step 3, estimate user weighted rate and select the users with the largest estimated weighted rate during this round of selection. Based on the orthogonal propagation domain $\widehat{\mathbf{D}}_{n}$, the estimated weighted rate of $n$-th users is formulated as:

$$
\widehat{R}_{\text {weighted }, n}=\mu_{n} \widehat{R}_{n}=\mu_{n} \log \left(1+\frac{P}{K}\left\|\widehat{\mathbf{D}}_{n}\right\|_{\mathrm{F}}^{2}\right),
$$

where $P$ is the downlink transmission power; $\|$.$\| stands for the Frobenius norm. The user$ with the largest weighted rate will be selected during the current round of selection. We denoted the selected user index as $s_{k}$ for $k$-th round of selection, where $s_{k} \in 1,2, \ldots, N$.

\footnotetext{
${ }^{2}$ Uplink CSI can be obtained from uplink transmission and for a wideband system, uplink CSI of one OFDM subcarrier only is enough.
} 
After selection, the selected user channel space will be updated by adding up the $s_{k}$-th user propagation domain as

$$
\widehat{\mathbf{S}} \leftarrow \widehat{\mathbf{S}}+\widehat{\mathbf{D}}_{s_{k}}^{H}\left(\widehat{\mathbf{D}}_{s_{k}} \widehat{\mathbf{D}}_{s_{k}}^{H}\right)^{-1} \widehat{\mathbf{D}}_{s_{k}} .
$$

The key part of zero-measurement selection is to estimate the weighted rate of each user in Step 3. Based on the findings that uplink-inferred downlink user channel norm and inter-user channel correlation bring in small amount of estimation error; as a result, the estimated channel norm project to the complementary space of selected user channel space will be close to actual downlink channel norm as

$$
\left\|\widehat{\mathbf{C}}_{n}(\mathbf{I}-\widehat{\mathbf{S}})\right\| \approx\left\|\mathbf{h}_{\operatorname{Dn}}(\mathbf{I}-\widehat{\mathbf{S}})\right\|
$$

where $\mathbf{h}_{D n}$ is the downlink channel of $n$-th user. Therefore, the estimated weighted sum rate $\widehat{R}_{\text {weighted, } n}$ will be close to the actual one and zero-measurement selection let the base-station select users based on the approximated weighted sum rate. Although there will still certain performance gap between zero-measurement selection and full-channel selection, which will be evaluated in the next section.

Combining the above three main steps, the zero-measurement selection algorithm is summarized as follows:

Input: Uplink CSIs of all the $N$ users $\hat{\mathbf{h}}_{\mathrm{Un}} \in \mathbb{C}^{M}, n=1,2, \ldots, N$.

Output: $\quad$ Selected user set $\mathcal{S}$, where $|\mathcal{S}|=K$.

Step 1:First extract uplink dominant path angles and amplitudes and construct estimated downlink dominant angle vectors $\widehat{\mathbf{C}}_{n}, n=1,2, \ldots, N$ for all the $N$ user using definition in Eq. 6. Then initialize the remaining user set as $\mathcal{T}=\{1,2, \ldots, N\}$, selected user set as $\mathcal{S}=\phi$, selected user channel space as $\widehat{\mathbf{S}}=\mathbf{0}_{M \times M}$, loop index as $i=1$.

Step 2: For all users $n \in \mathcal{T}$, calculate the orthogonal component $\widehat{\mathbf{D}}_{n}$ as in Eq. 11.

Step 3: Select user $s_{i}$ that has the largest weighted rate as

$$
s_{i}=\arg \max _{\mathrm{n} \in \mathcal{T}} \mu_{\mathrm{n}} \log \left(1+\frac{\mathrm{P}}{\mathrm{K}}\left\|\widehat{\mathbf{D}}_{\mathrm{n}}\right\|_{\mathrm{F}}^{2}\right)
$$

then update selected user set as $\mathcal{S} \leftarrow \mathcal{S} \cup\left\{s_{i}\right\}$, selected user channel space as

$$
\widehat{\mathbf{S}} \leftarrow \widehat{\mathbf{S}}+\widehat{\mathbf{D}}_{s_{i}}^{H}\left(\widehat{\mathbf{D}}_{s_{i}} \widehat{\mathbf{D}}_{s_{i}}^{H}\right)^{-1} \widehat{\mathbf{D}}_{s_{i}}
$$

remaining user set as

$$
\mathcal{T}=\left\{n \in \mathcal{T}, k \neq s, \frac{\left\|\widehat{\mathbf{C}}_{n} \widehat{\mathbf{D}}_{(s)}^{H}\left(\widehat{\mathbf{D}}_{(s)} \widehat{\mathbf{D}}_{(s)}^{H}\right)^{-1} \widehat{\mathbf{D}}_{(s)}\right\|}{\left\|\widehat{\mathbf{C}}_{n}\right\|}<\alpha\right\}
$$

where $\alpha$ is a small positive constant set to remove users with large correlation to the selected user, and loop index as $i \leftarrow i+1$. If $i<K$, go to Step 2. Otherwise the algorithm is finished. 


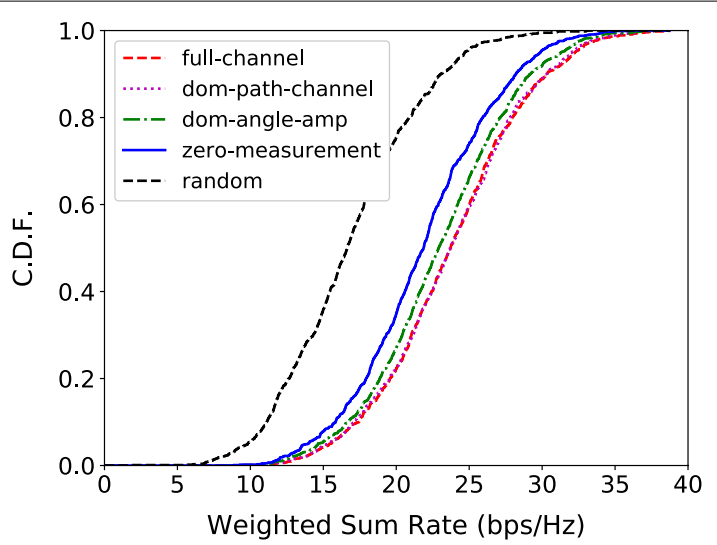

Fig. 5 The cumulative distribution function of weighted sum rate. This includes weighted sum rates with full-channel selection (labeled as full channel), dominant channel paths-based selection (labeled as dom-path-channel), dominant angles and amplitudes-based selection (labeled as dom-angle-amp), zero-measurement selection (labeled as zero-measurement) and uniformly random user selection (labeled as random), respectively, where a 64 -antenna base-station selects $K=8$ users out of $N=100$ users for downlink beamforming based on measured channels

\section{Results and discussion}

In this section, we evaluate the performance of zero-measurement selection. We first employ measured FDD massive MIMO channels to evaluate the performance of zeromeasurement selection by comparing to full channel-based selection and then examine the scalability with both the number of base-station antennas and the number of users for zero-measurement selection. Then, for further performance validation, we employ the 3GPP spatial channel model to evaluate the performance and examine the scalability for zero-measurement selection.

\subsection{Experimental results}

The details of measured channels are shown in [14]. Since the goal of downlink user selection is to maximize the weighted sum rate of $K$ selected users, as shown in Eq. 10, we take the weighted sum rate of selected users as the key metric to evaluate the performance of zero-measurement selection.

For comparison, we implement the full-channel selection [18], which assumes perfect downlink CSIs of all the $N$ users are available. To observe the performance gap between zero-measurement selection and full-channel selection, we consider the system where a 64-antenna base-station selects $K=8$ users out of $N=100$ users for downlink. The cumulative distribution function of weighted sum rate based on 1000 cases is shown in Fig. 5. In each case, the 100 users' uplink and downlink channels are randomly selected from the measured channel dataset and the weights are assumed i.i.d with $U(0,1]$ for simplicity. Then, we set different numbers of antennas and different numbers of users to evaluate the performance scalability for zero-measurement selection.

Finding 3 - Zero-measure Selection Performs Close to Full-channel Selection: From Fig. 5, we can observe that zero-measure selection achieves average $92.5 \%$ weighted 


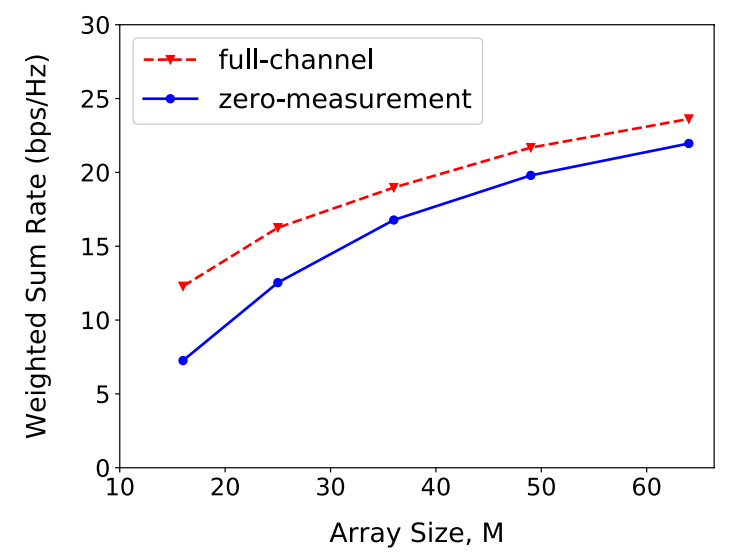

Fig. 6 The weighted sum rate with zero-measurement selection and full-channel selection, respectively. The system setup is that a changing number of antennas base-station select 4 users out of 100 users for downlink beamforming based on measured channels

sum rate of full-channel selection one. While in terms of overhead, $O(N=100)$ downlink channel estimation overhead incurs to obtain full downlink channel, while no channel estimation incurs for zero-measure selection and only $O(K=8)$ channel estimation overhead is needed to perform effective beamforming to selected $K$ users.

Explanation for Finding 3: To understand the performance gap between zero-measure selection and full-channel selection, we also implement two other user selection schemes, one is user selected based on downlink dominant paths approximated channel, and the other one is user selection based on downlink dominant angle vectors. We find out that the main source of performance gap comes from the missing dominant path phase information (3.5\%) and dominant angles with amplitudes mismatch effect (4\%). For the missing dominant path phase information effect, without phase information, the estimated user weighted rate will be smaller than the actual one, as explained in the previous section. For the dominant angles with amplitudes mismatch effect, both inferred inter-user channel correlation and user channel norm from uplink will have estimation error and thus affect zero-measure selection performance.

Finding 4 - Zero-measure Selection Scales with Base-station Array Size: From Fig. 6, we can observe that the weighted sum rate based on zero-measurement selection increases with the number of base-station antennas, from $7.25 \mathrm{bps} / \mathrm{Hz}$ with 16 antennas to $22 \mathrm{bps} / \mathrm{Hz}$ with 64 antennas. Compared to full-channel selection, zero-measurement selection achieves a larger relative weighted sum rate with more base-station antennas, from 59\% with 16 antennas to about $93 \%$ with 64 antennas. Besides, the weighted sum rate gap between zero-measurement selection and full-channel selection decreases with the number of base-station antennas, from $5.05 \mathrm{bps} / \mathrm{Hz}$ with 16-antenna to $1.6 \mathrm{bps} / \mathrm{Hz}$ with 64-antenna. A similar trend is also shown in Fig. 8.

Explanation for Finding 4: The main source of zero-measurement selection performance loss comes from the missing dominant path phase effect and dominant angles with amplitudes' mismatch effect, while both effects will have a smaller impact on zero-measurement selection with more base-station antennas. As a result, the 


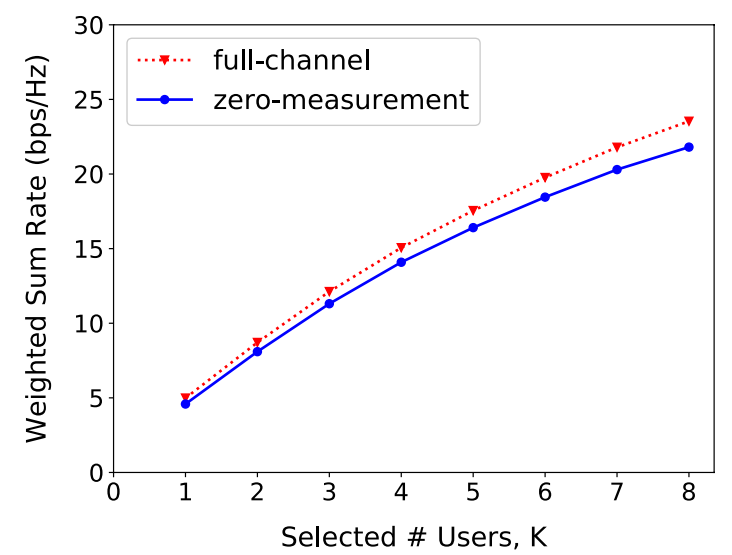

Fig. 7 The weighted sum rate with zero-measurement selection and full-channel selection, respectively. The system setup is that a 64-antenna base-station select changing number of users out of 100 users for downlink beamforming based on measured channels

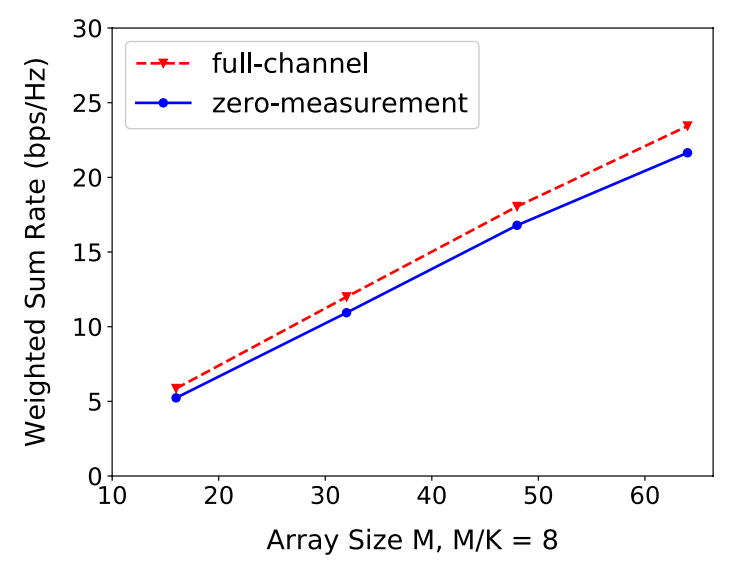

Fig. 8 The weighted sum rate with zero-measurement selection and full-channel selection, respectively. The system setup is that a changing number of antennas select changing number of users with fixed ratio as 8 out of 100 users for downlink beamforming based on measured channels

weighted sum rate gap between zero-measurement selection and full-channel selection decreases with the number of base station.

Finding 5 - Zero-measure Selection Scales with the Number of Selected Users: For the scalability with the number of selected users, from Fig. 7, we can observe that the weighted sum rate based on zero-measurement selection increases with the number of selected users, from $8.1 \mathrm{bps} / \mathrm{Hz}$ with 2 users to $21.8 \mathrm{bps} / \mathrm{Hz}$ with 8 users. While the weighted sum rate gap compared to full-channel selection increases slightly with the number of selected users, from $0.6 \mathrm{bps} / \mathrm{Hz}$ with 2 users to $1.7 \mathrm{bps} / \mathrm{Hz}$ with 8 users. A similar trend is also shown in Fig. 8.

Explanation for Finding 5: Since both the missing path phase information effect and dominant angles with amplitudes mismatch effect exist for each selected user, the weighted sum rate gap between zero-measurement selection and full-channel selection increases with the number of selected users. 


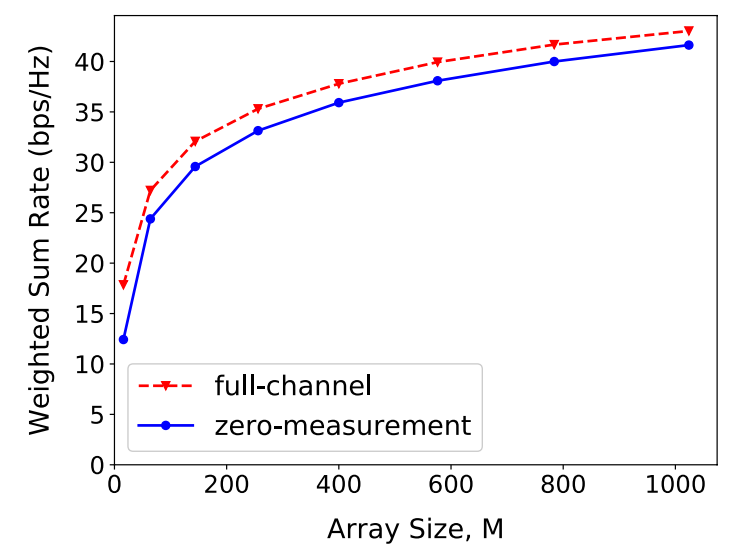

Fig. 9 The weighted sum rate with zero-measurement selection and full-channel selection, respectively. The system setup is that a changing number of antennas base-station select 4 users out of 100 users for downlink beamforming based on simulated channels

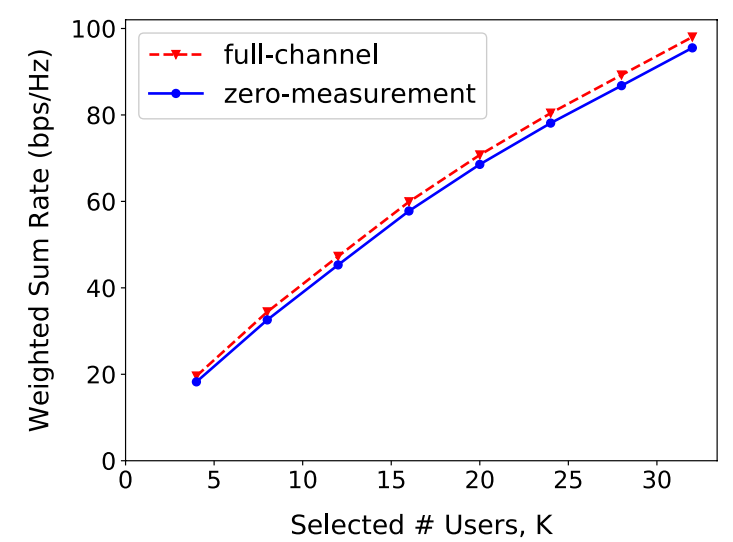

Fig. 10 The weighted sum rate with zero-measurement selection and full-channel selection, respectively. The system setup is that a 64-antenna base-station selects changing number of users out of 100 users for downlink beamforming based on simulated channels

\subsection{Numerical results}

To further validate experimental findings on zero-measurement selection in a larger array regime, we employ the 3GPP spatial channel model [29], to evaluate the performance of zero-measurement selection. The system setup here is similar to experimental setup, and the results are shown in Figs. 9, 10, and 11.

First, from Fig. 9, we can observe that zero-measure selection performs close to fullchannel selection, achieving an average $90 \%$ weighted sum rate with 64 antennas at the base station, which matches Finding 3. For the scalability with the base-station array size, we can observe that the weighted sum rate based on zero-measurement selection increases with the number of base-station antennas, from $12.4 \mathrm{bps} / \mathrm{Hz}$ with 16 antennas to $41.6 \mathrm{bps} / \mathrm{Hz}$ with 1024 antennas, and the weighted sum rate gap compared to fullchannel selection decreases with the number of base-station antennas, which matches Finding 4 in a larger number of base-station antennas regime. 


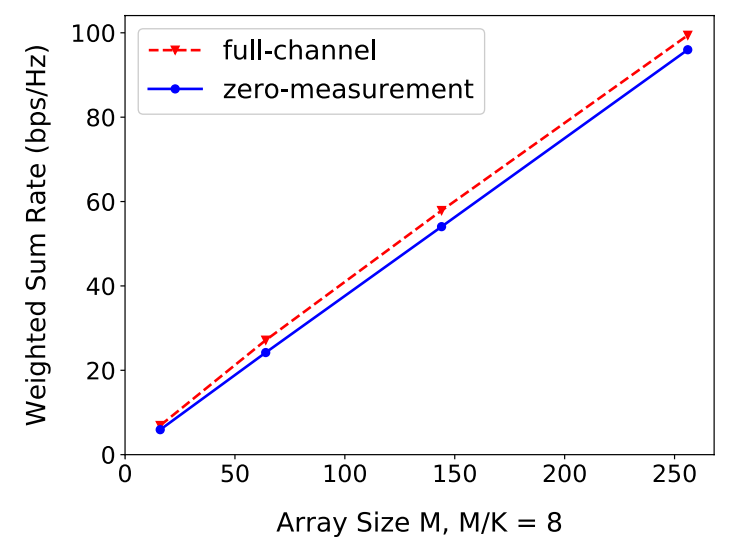

Fig. 11 The weighted sum rate with zero-measurement selection and full-channel selection, respectively. The system setup is that where a changing number of antennas select changing number of users with fixed ratio as 8 out of 100 users for downlink beamforming based on simulated channels

Second, for the scalability with the number of selected users, from Fig. 10, we can observe that the weighted sum rate based on zero-measurement selection increases with the number of selected users, from $18.3 \mathrm{bps} / \mathrm{Hz}$ with 4 users to $95.5 \mathrm{bps} / \mathrm{Hz}$ with 32 users when the base-station is equipped with 256 antennas. While the weighted sum rate gap compared to full-channel selection increases slightly with the number of selected users, from $1.3 \mathrm{bps} / \mathrm{Hz}$ with 4 users to $2.5 \mathrm{bps} / \mathrm{Hz}$ with 32 user, and the trend matches Finding 5.

Third, we can observe the combined scalability with both the number of base-station antennas and the number of selected users from Fig. 11. In more detail, the ratio between the number of base-station antennas and the number of selected users is fixed as 8 . The results show that, compared to full-channel selection, zero-measurement selection achieves average $90 \%$ weighted sum rate when a 64 -antenna base station selects 8 users out of 100 users for downlink beamforming. And the weighted sum rate percentage goes up to $97 \%$ when a 256 -antenna base-station selects 32 users out of 100 users for downlink beamforming.

\section{Conclusions}

To sum up, motivated by the experimental findings that both uplink inferred downlink inter-user channel correlation and uplink inferred user channel norm provide good approximation with small errors, we develop zero-measurement selection for FDD massive MIMO systems. Zero-measurement selection utilizes the uplink channel in the propagation domain to estimate the weighted sum rate and selects users that maximize the weighted sum rate as in proportional-fair selection. Thus, no downlink channel estimation overhead incurs in zero-measurement selection.

Then, we evaluate zero-measurement selection with both measured and simulated FDD massive MIMO channels. The results show that zero-measurement selection performs close to full-channel-based user selection, with only $7.5 \%$ performance loss on average when a 64-antenna selects 4 users out of 100 users for downlink beamforming. 
Also, zero-measurement selection scales well with both the number of base-station antennas and the number of users, as shown in both experimental and numerical results.

In this paper, while we develop zero-measurement selection in the proportional-fair form, the selection scheme can be easily extended to other forms of user selection.

\section{Abbreviations}

FDD: Frequency-division duplexing; MIMO: Multi-input multi-output; ZMS: Zero-measurement selection; CSI: Channel state information.

Acknowledgements

Not applicable.

Authors' contributions

The authors contributed equally. Both authors read and approved the final manuscript.

\section{Funding}

The authors were partially supported by NSF Grant 1518916 and support from Qualcomm, Inc.

Availability of data and materials

Not applicable

\section{Declarations}

\section{Competing interests}

The authors declare that they have no competing interests.

Received: 13 March 2021 Accepted: 15 November 2021

Published online: 03 December 2021

\section{References}

1. T.L. Marzetta, Noncooperative cellular wireless with unlimited numbers of base station antennas. IEEE Trans. Wirel. Commun. 9(11), 3590-3600 (2010)

2. C. Shepard, H. Yu, N. Anand, E. Li, T. Marzetta, R. Yang, L. Zhong, Argos: practical many-antenna base stations, in Proceedings of the 18th Annual International Conference on Mobile Computing and Networking (ACM, 2012) pp. 53-64

3. E.G. Larsson, O. Edfors, F. Tufvesson, T.L. Marzetta, Massive MIMO for next generation wireless systems. arXiv preprint arXiv:1304.6690 (2013)

4. F. Rusek, D. Persson, B.K. Lau, E.G. Larsson, T.L. Marzetta, O. Edfors, F. Tufvesson, Scaling up MIMO: opportunities and challenges with very large arrays. IEEE Signal Process. Mag. 30(1), 40-60 (2013)

5. H.Q. Ngo, E.G. Larsson, T.L. Marzetta, Energy and spectral efficiency of very large multiuser MIMO systems. IEEE Trans. Commun. 61(4), 1436-1449 (2013)

6. C.-S. Lee, M.-C. Lee, C.-J. Huang, T.-S. Lee, Sectorization with beam pattern design using $3 \mathrm{~d}$ beamforming techniques, in 2013 Asia-Pacific Signal and Information Processing Association Annual Summit and Conference (IEEE, 2013), pp. 1-5

7. X. Cheng, B. Yu, L. Yang, J. Zhang, G. Liu, Y. Wu, L. Wan, Communicating in the real world: $3 d$ MIMO. IEEE Wirel. Commun. 21(4), 136-144 (2014)

8. L. You, X. Gao, X.-G. Xia, N. Ma, Y. Peng, Pilot reuse for massive MIMO transmission over spatially correlated Rayleigh fading channels. IEEE Trans. Wirel. Commun. 14(6), 3352-3366 (2015)

9. H. Xie, F. Gao, S. Zhang, S. Jin, A unified transmission strategy for TDD/FDD massive MIMO systems with spatial basis expansion model. IEEE Trans. Veh. Technol. 66(4), 3170-3184 (2016)

10. P. Patcharamaneepakorn, S. Wu, C.-X. Wang, M.M. Alwakeel, X. Ge, M. Di Renzo et al., Spectral, energy, and economic efficiency of $5 \mathrm{~g}$ multicell massive MIMO systems with generalized spatial modulation. IEEE Trans. Veh. Technol. 65(12), 9715-9731 (2016)

11. N. Garcia, H. Wymeersch, E.G. Larsson, A.M. Haimovich, M. Coulon, Direct localization for massive MIMO. IEEE Trans. Signal Process. 65(10), 2475-2487 (2017)

12. X. Du, A. Sabharwal, Shared angles-of-departure in massive MIMO channels: correlation analysis and performance impact. IEEE Trans. Wirel. Commun. 6, 66 (2019)

13. X. Du, Y. Sun, N. Shroff, A. Sabharwal, Balance queueing and retransmission: latency-optimal massive MIMO design. arXiv preprint arXiv:1902.07676 (2019)

14. X. Zhang, L. Zhong, A. Sabharwal, Directional training for FDD massive MIMO. IEEE Trans. Wirel. Commun. 6, 66 (2018)

15. P. Viswanath, D.N.C. Tse, R. Laroia, Opportunistic beamforming using dumb antennas. IEEE Trans. Inf. Theory 48(6), 1277-1294 (2002)

16. H. Viswanathan, K. Kumaran, Rate scheduling in multiple antenna downlink wireless systems. IEEE Trans. Commun. 53(4), 645-655 (2005)

17. Z. Shen, R. Chen, J.G. Andrews, R.W. Heath, B.L. Evans, Low complexity user selection algorithms for multiuser MIMO systems with block diagonalization. IEEE Trans. Signal Process. 54(9), 3658-3663 (2006) 
18. T. Yoo, A. Goldsmith, On the optimality of multiantenna broadcast scheduling using zero-forcing beamforming. IEEE J. Sel. Areas Commun. 24(3), 528-541 (2006)

19. T. Ji, C. Zhou, S. Zhou, Y. Yao, Low complex user selection strategies for multi-user MIMO downlink scenario, in 2007 IEEE Wireless Communications and Networking Conference (IEEE, 2007), pp. 1532-1537

20. A. Bayesteh, A.K. Khandani, On the user selection for MIMO broadcast channels. IEEE Trans. Inf. Theory 54(3), 1086-1107 (2008)

21. S. Sigdel, W.A. Krzymien, Simplified fair scheduling and antenna selection algorithms for multiuser MIMO orthogonal space-division multiplexing downlink. IEEE Trans. Veh. Technol. 58(3), 1329-1344 (2008)

22. J. Wang, D.J. Love, M.D. Zoltowski, User selection with zero-forcing beamforming achieves the asymptotically optimal sum rate. IEEE Trans. Signal Process. 56(8), 3713-3726 (2008)

23. J. Mao, J. Gao, Y. Liu, G. Xie, Simplified semi-orthogonal user selection for MU-MIMO systems with ZFBF. IEEE Wirel. Commun. Lett. 1(1), 42-45 (2012)

24. K. Ko, J. Lee, Multiuser MIMO user selection based on chordal distance. IEEE Trans. Commun. 60(3), 649-654 (2012)

25. S. Huang, H. Yin, J. Wu, V.C. Leung, User selection for multiuser MIMO downlink with zero-forcing beamforming. IEEE Trans. Veh. Technol. 62(7), 3084-3097 (2013)

26. M. Benmimoune, E. Driouch, W. Ajib, D. Massicotte, Joint transmit antenna selection and user scheduling for massive MIMO systems, in 2015 IEEE Wireless Communications and Networking Conference (WCNC) (IEEE, 2015), pp. 381-386

27. C. Chen, W. Cai, X. Cheng, L. Yang, Y. Jin, Low complexity beamforming and user selection schemes for $5 \mathrm{~g}$ MIMONOMA systems. IEEE J. Sele. Areas Commun. 35(12), 2708-2722 (2017)

28. H. Krim, M. Viberg, Two decades of array signal processing research: the parametric approach. IEEE Signal Process. Mag. 13(4), 67-94 (1996)

29. 3GPP: Study on 3D channel model for LTE. TR 36.873, 3rd Generation Partnership Project (3GPP) (June 2015). http:// www.3gpp.org/dynareport/36873.htm

\section{Publisher's Note}

Springer Nature remains neutral with regard to jurisdictional claims in published maps and institutional affiliations.

\section{Submit your manuscript to a SpringerOpen ${ }^{\odot}$ journal and benefit from:}

- Convenient online submission

- Rigorous peer review

- Open access: articles freely available online

- High visibility within the field

- Retaining the copyright to your article

Submit your next manuscript at $\gg$ springeropen.com 